\title{
Intraductal Papillary Mucinous Neoplasm Presenting as Bleeding Duodenal Mass: A Surgical Rarity
}

\author{
Nornazirah Azizan ${ }^{a}$, Firdaus Hayati ${ }^{b}$, Andee Dzulkarnaen Zakariac ${ }^{c}$, Nordashima Abd Shukor ${ }^{d}$ \\ aDepartment of Pathobiology and Medical Diagnostic, Faculty of Medicine and Health Sciences, Universiti \\ Malaysia Sabah, Sabah, Malaysia \\ bepartment of Surgery, Faculty of Medicine and Health Sciences, Universiti Malaysia Sabah, Sabah, Malaysia \\ 'Department of Surgery, School of Medical Sciences, Universiti Sains Malaysia, Kelantan, Malaysia \\ ${ }^{\mathrm{d} H i s t o p a t h o l o g y}$ Unit, Department of Pathology, Universiti Kebangsaan Malaysia, Kuala Lumpur, Malaysia
}

\section{ABSTRACT}

Intraductal papillary mucinous neoplasm (IPMN) is a rare pancreatic neoplasm. The presentation varies from recurrent pancreatitis, steatorrhea and weight loss to incidental findings during imaging studies. The recognition of IPMN is crucial in deciding for prompt surgical intervention, which is the best treatment modality for this precancerous condition. Here, we report a case of 55-year-old man with massive upper gastro intestinal bleeding arising from a huge fungating duodenal mass. In view of massive bleeding, a decision for emergency Whipple's pancreaticoduodenectomy was made. Final histological diagnosis confirmed as IPMN. To the best of our knowledge, this is the first case of IPMN presented with a huge fungating duodenal mass causing massive UGIB requiring surgical intervention.

KEYWORDS: intraductal papillary mucinous neoplasm, upper gastro intestinal bleeding, fungating duodenal mass, pancreatic invasive carcinoma

\section{INTRODUCTION}

Intraductal papillary mucinous neoplasm (IPMN) is a rare cystic neoplasm of the pancreas. It accounts for $1 \%$ of exocrine pancreatic tumour. ${ }^{1}$ It is a precursor for pancreatic invasive carcinoma, thus showing neoplastic progression from a benign intraductal tumour through increasing grades of dysplasia to invasive adenocarcinoma. ${ }^{2}$ Many literatures have described the variable presentations of IPMN, which include recurrent pancreatitis, deterioration of diabetes mellitus, steatorrhea, and weight loss to pancreatic incidentaloma ${ }^{3}$. However, none of these literatures describe upper gastro intestinal bleeding (UGIB) as part of IPMN presentations.

The recognition of IPMN requires various modalities including imaging studies,

Corresponding Author:

Dr. Firdaus Hayati, Department of Surgery, Faculty of Medicine and Health Sciences, Universiti Malaysia Sabah, Sabah, Malaysia

Tel : 088-320000 ext. 611073

E-mail: firdaushayati@gmail.com oesophagogastroduodenoscopy (OGDS) and subsequently confirmed by histology. We report an interesting case of an IPMN presented with massive UGIB requiring emergency Whipple's pancreaticoduodenectomy (WP) and histological examination revealed a duodenal mass arising from main pancreatic duct extending into duodenal lumen.

\section{CASE REPORT}

A 55-year-old gentleman presented with dizziness, palpitation and near fainting, which brought him to the hospital. Upon presentation, he was pale with class 3 hypovolemic shock. He was transfused with 10 pints of packed cells with 3 cycles of disseminated intravascular coagulopathy regime within 24 hours. An OGDS was performed which revealed a tumour at the ampulla of Vater (Figure 1). Computed tomography (CT) after stabilization showed a large heterogeneous polypoidal mass extending from level 2 (D2) of duodenum down to proximal D3 region causing partial obstruction of the duodenum and biliary system (Figure 2a). The pancreatic duct was ectatic at the pancreatic body (Figure 2b). However, no metastatic lesion noted. In 
view of ongoing bleed, an emergency WP was performed.
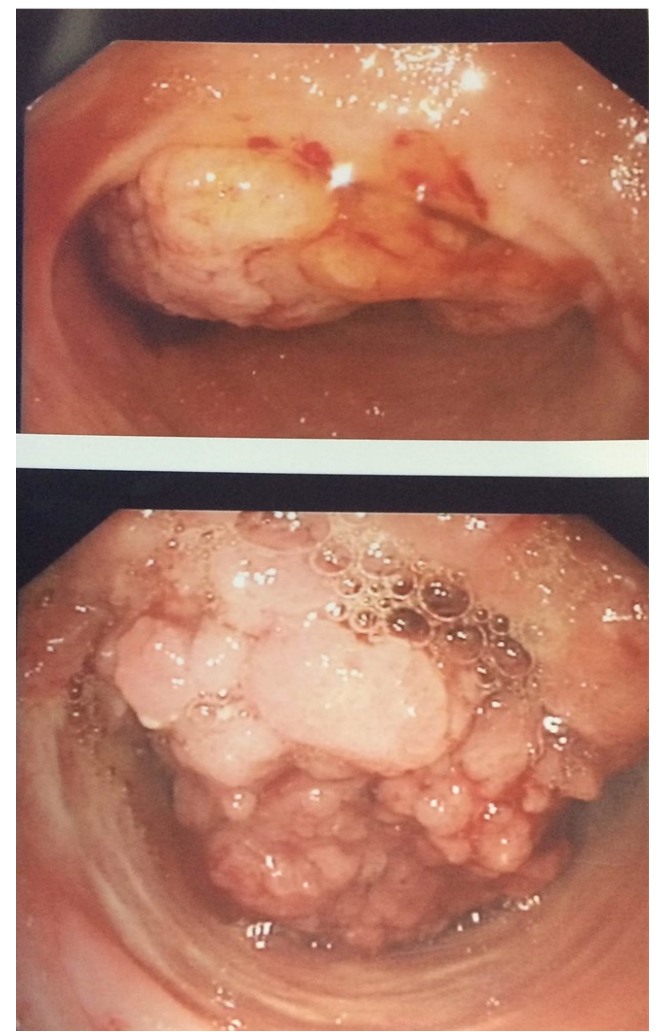

Figure 1: Endoscopic picture of duodenum showing a pedunculated growth with mucin excretion

The gross specimen revealed a huge pedunculated mass occupying level 2 and 3 duodenum measuring $125 \mathrm{~mm} \times 70 \mathrm{~mm} \times 60 \mathrm{~mm}$ (Figure 3 ). The mass was noted arising from the pancreatic duct extending into the duodenum obscuring the ampulla of Vater and causing partial obstruction of the duodenum. Cut section of the tumour showed whitish-mucoid cut surface.

Histopathological examination confirmed IPMN with low-grade dysplasia. The lesion was a large viloglandular polypoidal mass arising from the main pancreatic duct and extending into the duodenal mucosa, composed of compact glands forming papillary and pseudopapillary structure lined by tall columnar mucin-containing epithelial cells with thick fibrovascular core (Figure 4a).

The lining epithelium was generally bland looking. In areas, the epithelial cells showed evidence of low-grade dysplasia with pseudostratified enlarged nuclei with coarse chromatin pattern and some cells with prominent nucleoli (Figure 4b). The tumour was pushing and compressing against the duodenal wall but no stromal invasion seen. Immunohistochemical studies showed the cells are positive for CK7 and CK19 and negative for CK20.

Patient was treated postoperatively with standard enhanced recovery after surgery protocol and was discharged home without any complication ${ }^{4}$.

\section{DISCUSSION}

IPMN is first reported as early as in 1982 which described 'mucin-producing' pancreatic growth in four patients. ${ }^{5}$ The incidence of IPMN is climbing in recent years in view of improved awareness towards usage of imaging modalities in asymptomatic individuals. As a precursor of invasive carcinoma, it carries a poor prognosis if neglected and is overwhelmingly lethal if early diagnosis is not promptly established ${ }^{2}$.

Common presentations include abdominal pain, nausea and vomiting, weight loss, steatorrhea, and jaundice. They may have underlying recurrent pancreatitis and new onset or deterioration of diabetes mellitus. Traditionally, IPMN will not present with anaemia or features of malignancy. No mass or ascites can be felt per abdomen unless in advanced pancreatic cancer.

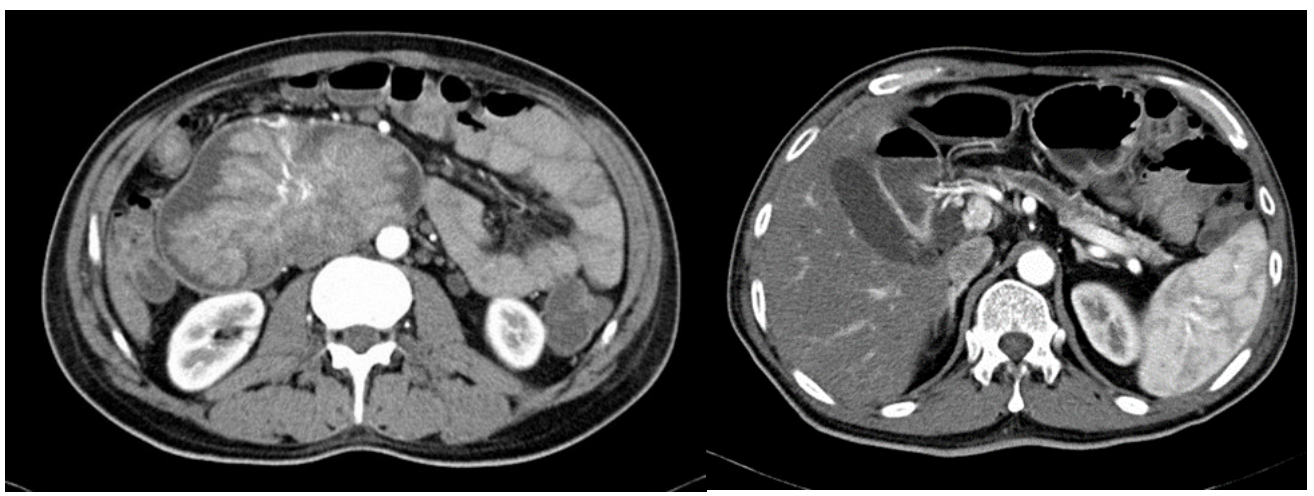

Figure 2a: Axial CT showing a heterogeneous duodenal mass. Figure 2b: Dilated pancreatic duct (arrow) 


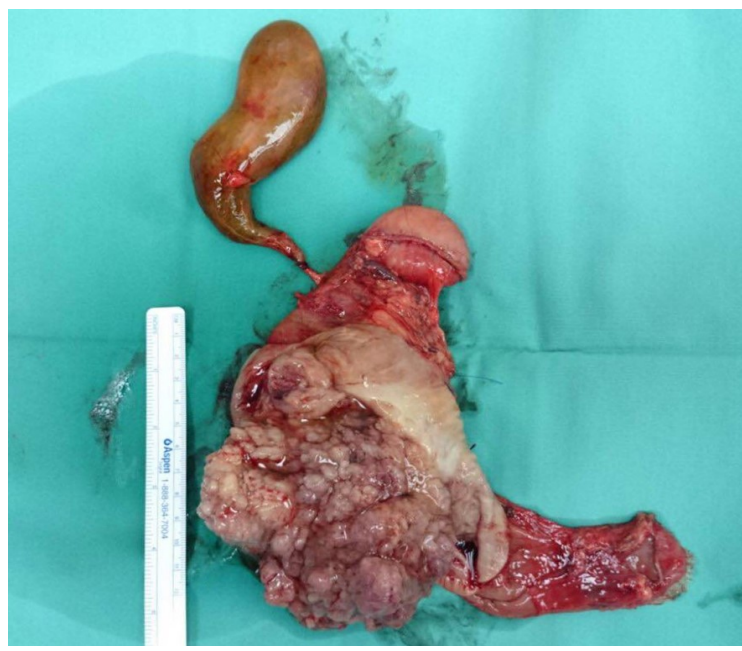

Figure 3: Whipple's procedure specimen revealed a malignant looking growth with mucin production arising from ampulla of Vater

Liver function test will be abnormal especially in biliary obstruction, causing raised direct bilirubin and alkaline phosphatase. Serum oncoproteins or tumour markers, such as carcinoembryonic antigen (CEA) and CA 19-9 level, are usually normal unless the IPMN is associated with invasive cancer. ${ }^{6}$

OGDS is crucial to assess stigmata of bleeding so intervention can follow. The finding of mucin extruding from the ampulla of Vater is classical of IPMN. ${ }^{6}$ Endoscopic retrograde cholangiopancreatography (ERCP) demonstrates a dilated pancreatic duct and filling defects, caused by intraluminal mucous plugs or papillary projections of the neoplasm itself. $^{6}$ Endoscopic ultrasound meanwhile helps by getting fluid aspiration for CEA and amylase, whereby both will be elevated.

CT scan in pancreatic protocol generally is important for the staging purposes. Apart from looking for locally advanced disease such as portal vein and inferior vena cava involvement, it is important to look for metastatic disease such as liver metastasis and ascites. In IPMN, CT usually reveals a dilated main pancreatic duct or a collection of cysts that represent dilated branch ducts. ${ }^{6}$ Magnetic resonance cholangiopancreatography may have a role but it depends on the necessity of the facility. It may demonstrate ductal dilatation and mural nodules. ${ }^{6}$ However, it is time-consuming especially in emergency situation.

Macroscopically, IPMN appears as a dilatation of the main duct or as cysts communicated with the excretory duct system producing grossly visible intraductal finger-like papillary projection with more than $1 \mathrm{~cm}$ in size. ${ }^{7,8}$ Any solid lesion in IPMN should be suspected of invasive carcinoma. Duodenal adenocarcinoma, head of pancreas carcinoma and solid-pseudopapillary carcinoma of the pancreas with duodenal extension are the mainstay diagnoses that should be considered. ${ }^{9,10}$ IPMN is characterized histologically by intraductal proliferation of columnar mucin-producing cells. It is of pancreatic in origin. The epithelium can be flat, simple villouslike or complex branching papillae with fibrovascular cores. The underlying stroma shows a conventional fibrous tissue, which by definition cannot be of ovarian type, as seen in mucinous cystic neoplasm ${ }^{7}$. IPMN can be categorized into main-duct type, branch -duct type and oncocytic type. ${ }^{11}$ In this case, there is a solid huge mass within the duodenum with no obvious pancreatic cystic lesion seen given an impression of duodenal adenocarcinoma from the gross appearance. Microscopically there is no evidence of invasion identified despite thorough sampling given a final diagnosis of IPMN (main-duct type).

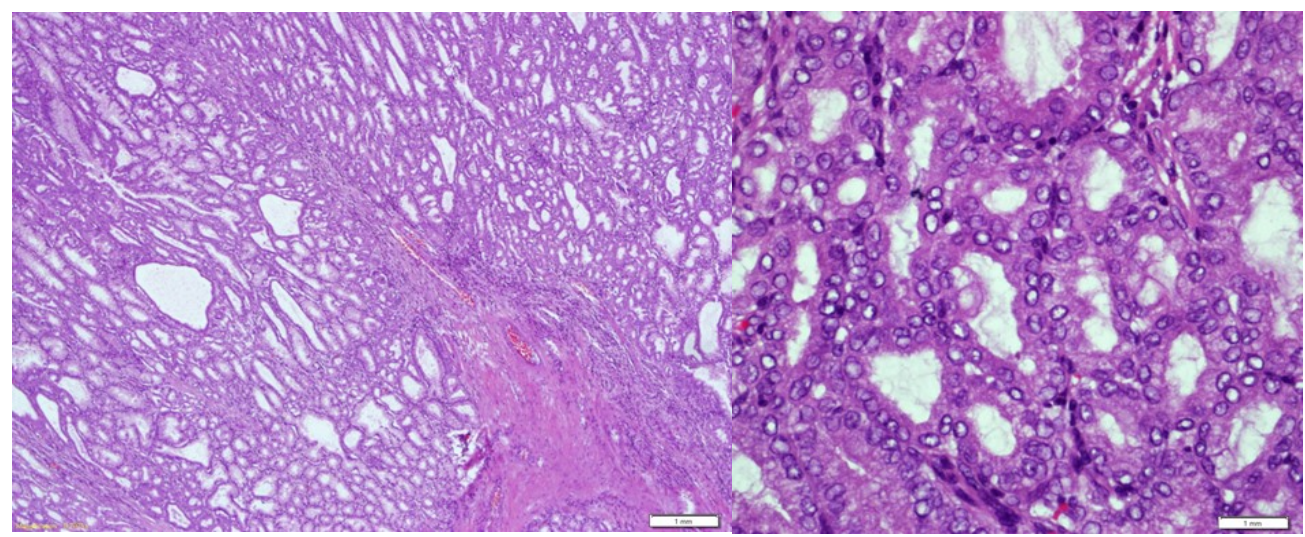

Figure 4a: The tumour is composed of compact glands forming papillary structures lined by tall columnar mucincontaining epithelial cells with thick fibrovascular core (Haematoxylin \& Eosin, 4x). Figure 4b: Higher magnification area of low-grade dysplasia showing pseudostratified enlarged nuclei with coarse chromatin pattern and some cells with prominent nucleoli. (Haematoxylin \& Eosin, 40x) 


\section{CONCLUSION}

Bleeding duodenal mass is an unusual presentation of IPMN as no literature described regarding this. Extensive sampling and thorough microscopic evaluation are crucial to look for evidence of invasion. Following this, better outcome and prognosis can be ascertained in IPMN rather than in invasive carcinoma.

\section{REFERENCES}

1. Gru "tzmann R, Niedergethmann M, Pilarsky C, Kloppel G, Saeger HD. Intraductal Papillary Mucinous Tumors of the Pancreas: Biology, Diagnosis, and Treatment. The Oncologist 2010;15:1294-1309

2. Wolfgang $\mathrm{CL}$, Herman JM, Laheru DA, Klein AP, Erdek MA, Fishman EK, Hruban RH. Recent progress in pancreatic cancer. CA Cancer J Clin 2013;63:318-348

3. Minagawa N, Sato N, Mori Y, Tamura T, Higura A, Yamaguchi $\mathrm{K}$. A comparison between intraductal papillary neoplasms of the biliary tract (BTIPMNs) and intraductal papillary mucinous neoplasms of the pancreas (P-IPMNs) reveals distinct clinical manifestations and outcomes. Eur J Surg Oncol 2013;39:554-558

4. Xiong J, Szatmary $P$, Huang $W$, de la IglesiaGarcia D, Nunes QM, Xia Q, Hu W, Sutton R, Liu $X$, Raraty MG. Enhanced Recovery After Surgery Program in Patients Undergoing Pancreaticoduodenectomy: A PRISMA-Compliant Systematic Review and Meta-Analysis. Medicine (Baltimore) 2016;95:e3497

5. Ohasi K, Murakami Y, Maruyama M. Four cases of mucin-producing cancer of the pancreas on specific findings of the papilla of Vater. Prog Dig Endoscopy 1982;20:348-351

6. Hruban, RH.; Pitman, MB.; Klimstra, DS. Atlas of tumor pathology. Fascicle 6. Washington, DC: American Registry of Pathology and Armed Forces Institute of Pathology; 2007. Tumors of the pancreas. Fourth

7. Victor MC, Carolina IA, Guadalupe LA, Francisco CR. Pathological features and diagnosis of intraductal papillary mucinous neoplasm of the pancreas. World Journal of Gasyrointestinal Oncology 2014;6:311-324

8. Tanaka M, Fernández-del Castillo C, Adsay V, Chari S, Falconi M, Jang JY, Kimura W, Levy P, Pitman MB, Schmidt CM, Shimizu M, Wolfgang $\mathrm{CL}$, Yamaguchi K, Yamao K. International consensus guidelines 2012 for the management of IPMN and MCN of the pancreas. Pancreatology 2012;12:183-197

9. Usuda D, Hashimoto Y, Muranaka E, Okamura H, Kanda T, Urashima S. Primary Duodenal Adenocarcinoma without Stenosis: A Case Report with a Brief Literature Review. Case Rep Oncol 2014;7:444-451

10. Mat ZAA, Shakir KA, Aminuddin AR, Mahedzan MR, Irnawati WA, Andee DZ, Hassan SA, Ezane MA, Hasnan MN. Solid-pseudopapillary carcinoma: a case study and literature review. BMJ Case Rep. 2012; Aug 27;2012

11. Bosman FT, Carneiro F, Hruban RH, Theise ND. WHO Classification of Tumours of Digestive System. IARC: Lyon 2010 